\title{
Fibrosarcoma of the chest wall: A case report
}

\author{
SF Kabir
}

\begin{abstract}
An 85 year old man presented with a large swelling over the right side of the chest wall of 3 years duration. Patient had no complaints other than a swelling which looked aggressive. On the 1st instance it looked malignant. Histopathology of the specimen confirmed that it is a case of fibrosarcoma. Excision of the tumor done with $2 \mathrm{~cm}$ healthy margin and the patient was referred to radiotherapist for possible adjuvant therapy.
\end{abstract}

Introduction: Fibrosarcoma is the most common primary soft tissue cancer of the chest wall. It occurs most frequently in young adults. The incidence of fibrosarcoma is about 0.05 cases per 100,000 population per year. ${ }^{1}$ Male and female are equally affected. More than half of the cases occur in the long bones, the distal femur and proximal tibia are the commonest sites. The humerus and scapula together have been reported as representing perhaps the second or third most common site. These tumors have been reported in every decade but are most common in the third and fourth decades. The signs and symptoms are those of pain and, at times, local swelling.

Case report: An 85 yrs Bangladeshi farmer from Noakhali, weighing $55 \mathrm{Kg}$, non smoker, non diabetic was admitted in a private clinic at Dhaka with the complaints of a large swelling on the right side of the anterior chest wall for 3 years. The swelling was gradually increasing in size but in the last three months the swelling had increased two fold according to the patient's statement. Other than the swelling he had no complaints. On examination, he was mildly anaemic, non ecteric, no lymph node enlargement, both axillary and neck regions were normal. Skin was free from the swelling. It is about $12.5 \mathrm{~cm} 10 \mathrm{~cm}$ in size, mobile from side to side and above downwards, not fixed to the underling muscle, the upper part of the swelling was ulcerated.

Laboratory investigations are within normal limit, X-ray chest and ECG were normal. Fine needle aspiration was positive for sarcomatous lesion.

Under general anesthesia the swelling was resected with $2 \mathrm{~cm}$ healthy margin. Wound was closed keeping a drain insitu. The histopathology report confirmed fibrosarcoma of moderate grading.

Discussion: Soft tissue sarcomas account for approximately $1 \%$ of all new cancer diagnosed. Slightly more than half of all patients diagnosed with the disease eventually die as a result of the cancer. Classically, soft tissue sarcomas present as asymptomatic large masses in the extremities or retroperitoneum, but they also develop with some frequency in the neck and within the abdominal viscera. Fifty percent of these tumors occur in the lower extremity, most commonly in the thigh. Soft tissue sarcomas originate from a wide variety of mesenchymal cell types and include liposarcoma, fibrosarcoma, rhabdomyosarcoma, and desmoids tumors. While the histopathology of these tumors is highly variable, with some exceptions they tend to behave in a fashion directed by tumor grade rather than the cell of origin.

Most soft tissue sarcomas arise de novo, and rarely do they result from malignant degeneration of a benign lesion. There are some familial syndromes in which patients are genetically predisposed to the formation of soft tissue sarcomas.

Any mass suspected of harboring a soft tissue sarcoma should be imaged prior to operation. Both CT and MRI can be used to image these tumors, but MRI is often more accurate in defining the extent of the tumor and invasion of surrounding structures. A chest X-ray or chest CT should be obtained in order to evaluate for pulmonary metastases in patients with high-grade tumors. In imaging a retroperitoneal tumor, the liver should be examined in the same radiographic study.

The most important prognostic variables for patients with soft tissue sarcoma are the size and grade of the primary tumor. ${ }^{2}$ As grading is based on the cellular architecture and invasive nature of the tumor FNA is not a typically useful biopsy technique for the initial diagnosis of a sarcoma. Core needle biopsies may be performed for large, palpable superficial tumors. For large deep tumors or those adjacent to vital structures, incisional biopsy is usually the diagnostic method of choice. If a tumor is small $(<3 \mathrm{~cm})$ and superficial, excisional biopsy should be performed.

Treatment: Surgery is the mainstay of therapy for sarcoma, but these tumors are also relatively sensitive to radiation ${ }^{3}$. A pseudocapsule composed of tumor cells surrounds sarcomas, and local invasion along fascial planes and neurovascular structures is common. Local capsular excision is inadequate for cure, as local recurrence rates for such procedures are greater than $90 \%$.

Generally, the margin for wide local excision should be at last $2 \mathrm{~cm}$. The dissection should be carried out at least one fascial plane away from the tumor and may necessitate a compartmental resection. The surgeon should take care to avoid direct visualization of the tumor during the procedure in order to prevent tumor from seeding into the wound. Surgical excision of the tumor may involve resection of important neurovascular structure.

Large soft tissue defects often require the construction of myocutaneous flaps to improve function and cosmesis. ${ }^{4}$ Soft tissue sarcomas rarely invade bone or skin, and wide 


\section{Fibrosarcoma of the chest wall}

resections of these structures are infrequently necessary. Following wide local excision, metal clips should be placed at all margins of the resection in order to guide subsequent radiotherapy.

Radiation therapy is a necessary adjunct in the local control of sarcomas. ${ }^{5}$ Based on the location and size of the tumor, several modes of delivery are used, including EBRT, intraoperative radiation, and brachytherapy. In many centers, small $(<2 \mathrm{~cm})$ low-grade extremity tumors are treated with wide local excision alone. Larger tumors should be treated by wide excision and postoperative radiation therapy, either by EBRT or brachytherapy catheters. Preoperative radiotherapy appears to have distinct advantages in patients with tumors larger than $10 \mathrm{~cm}$, improving local control rates and sometimes shrinking tumors sufficiently to allow for limb sparing procedures. ${ }^{6}$

Conclusion: The natural history is one of progressive growth, frequent pathologic fracture, and distant metastases usually to the lung, with death if appropriate treatment is not given. Treatment consists of local removal of the primary tumor, which in most situations requires amputation. Neither radiation therapy nor chemotherapy has been helpful. The 5 years survival rate is $30-35 \%$.

\section{Reference:}

1. Burt M. Primary malignant tumors of the chest wall. The memorial sloan kettering cancer center experience. Chest Suns Clin N arm, 1994;4:137.

2. Perry RR. Survival after surgical resection for high grade chest wall sarcoma. Ann Thorac Surg. 1990;49:363.

3. Brennan MF. The role of multimodality therapy in soft tissue sarcoma. Ann Surg. 1991;214:328.

4. Pisters PW. term results of a prospective Randomized trial of adjuvant brachytherapy in soft tissue sarcoma. J Clin Oncol. 1996;14:859.

5. Pennington DG, Marsdein W, Stephens FO. Fibrosarcoma of metacarpal treated by combination therapy and immediate reconstruction with vascularized bone graft. J Hand Surg An. 1991;16:877.

6. Sim FH, Frabosica FJ, Frassica DA. Soft tissue tumors: diagnosis, evaluation and management. Journal of the American Academy of Orthopedic Surgeon. 1994;2:202-211. 